\title{
Der Vergleich mit dem deutschen «Anordnungsmodell» hinkt gewaltig
}

\author{
Yvik Adler ${ }^{a}$, Stephan Wenger ${ }^{b}$ \\ ${ }^{a}$ lic. phil.; ${ }^{b}$ dipl. psych.; ${ }^{\text {a, b }}$ Co-Präsidenten der Föderation der Schweizer Psychologinnen und Psychologen (FSP)
}

Zum Thema dieses Beitrags siehe auch den nachfolgenden Artikel von Erich Seifritz.
Korrespondenz: Föderation der Schweizer Psychologinnen und Psychologen (FSP) fsp[at]fsp.psychologie.ch
Prof. Dr. Erich Seifritz nimmt im Artikel «Das Anordnungsmodell - Kopie eines 'kaputten Systems'» [1] Bezug auf das deutsche Modell der psychotherapeutischen Versorgung. Er vergleicht dieses direkt mit dem von der FSP geforderten Anordnungsmodell. Dieser Vergleich hinkt gewaltig. Das für die Schweiz geforderte Anordungsmodell hat mit dem deutschen Modell überhaupt nichts gemein.

Wie von Erich Seifritz beschrieben, hat der Arzt/die Ärztin im deutschen Modell lediglich die Aufgabe zu bescheinigen, dass «keine medizinische Kontraindikation für eine Psychotherapie besteht». Das ist im für die Schweiz geforderten Modell völlig anders. Was wir wollen, ist ein Modell, das demjenigen der Physiotherapie nachempfunden ist. Der Patient geht zur Ärztin, schildert seine Symptome, und die Ärztin ordnet aufgrund dieser Schilderung eine Psychotherapie an. Mit dieser Verordnung geht der Patient zu einer psychologischen Psychotherapeutin seiner Wahl. Die psychotherapeutische Behandlung wird dann von der Grundversicherung übernommen, wobei die Therapeutin direkt über die Grundversicherung abrechnet und nicht mehr wie im heutigen Delegationssystem über den delegierenden Arzt. Der Unterschied zum heutigen Modell ist also gar nicht so gross, wie von Seifritz insinuiert. Lediglich der Flaschenhals der Delegation wird beseitigt, was für alle Beteiligten und insbesondere für die Patientinnen und Patienten eine grosse Verbesserung darstellt. Verbesserung vor allem deshalb, weil neu alle Ärztinnen und Ärzte eine Psychotherapie verordnen können, nicht nur Fachärztinnen und Fachärzte für Psychiatrie und Psychotherapie. So genügt der Gang zum Hausarzt, um Zugang zu einer von der Grundversicherung bezahlten Psychotherapie zu erhalten.

Das heisst aber auch, dass der Arzt/die Ärztin weiterhin die Rolle des Gatekeepers übernimmt. Es ist an $\mathrm{ihm} / \mathrm{ihr}$ zu entscheiden, ob eine Psychotherapie angezeigt ist oder nicht. Auch die Qualitätssicherung wird mit dem Anordnungsmodell nicht vernachlässigt. Die entsprechenden Kontrollinstrumente müssen in der Verordnung definiert werden. So wird es zum Beispiel eine regelmässige Überprüfung der Psychotherapieindikation geben müssen.

Die konkrete Diagnosestellung und die Durchführung der Therapie können hingegen getrost den psychologischen Psychotherapeutinnen und -therapeuten überlassen werden. Diese sind anerkannte Fachleute, die nach fünf Jahren Masterstudium in Psychologie während weiterer vier Jahre eine eidgenössisch anerkannte Weiterbildung in Psychotherapie absolviert haben. Sie sind damit genauso gut ausgebildet wie Fachärztinnen und Fachärzte für Psychiatrie und Psychotherapie. Und sie sind qualifiziert, Diagnosen zu erstellen und Therapieverfahren auszuwählen und diese eigenständig durchzuführen. Sie tun dies auch bereits heute, entweder als Angestellte im Delegationsmodell oder wenn sie in eigener fachlicher Verantwortung arbeiten, mit einer von den Kantonen ausgestellten Berufsausübungsbewilligung.

Es ist deshalb nicht nachvollziehbar, wie Herr Seifritz auf die Idee kommt, dass psychologische Psychotherapeuten auf eine «fachärztliche Steuerung» angewiesen sind. Im Bundesgesetz über die Psychologieberufe, das seit sechs Jahren in Kraft ist und das auch die "Ausübung des Psychotherapieberufs» regelt, ist eine solche «Steuerung» jedenfalls nicht vorgesehen.

Die Gefahren, die Herr Seifritz in seinem Artikel heraufbeschwört, sehen wir nicht: Die «Kostenexplosion durch Mengenausweitung» wird es nicht geben, weil der ambulante Weg gestärkt wird. Wenn frühzeitig ambulant behandelt wird, können Chronifizierung verhindert und teure stationäre Behandlungen vermieden werden. Eine «Senkung des Psychiatrie-Tarifs» ist ganz bestimmt nicht im Interesse von uns psychologischen Psychotherapeutinnen und -therapeuten. Auch die Befürchtung einer «Unterversorgung durch Kostenverschiebung» ist völlig unbegründet. Die Ärztinnen und Ärzte haben es dank der Anordnungskompetenz selbst in der Hand, dieses Szenario zu verhindern.

\section{Literatur}

1 Seifritz E. Das Anordnungsmodell - Kopie eines «kaputten Systems». Schweiz Ärzteztg. 2019;100(15):540-1. DOI: https:// doi.org/10.4414/saez.2019.17757 